\title{
Solid-State Scrolls from Hierarchical Self-Assembly of T-Shaped Rod-Coil Molecules**
}

\author{
Dong-Je Hong, Eunji Lee, Haemi Jeong, Jeong-kyu Lee, Wang-Cheol Zin, Trung Dac Nguyen, \\ Sharon C. Glotzer, and Myongsoo Lee*
}

One-dimensional nanostructures such as cylinders and tubules are of great interest owing to their unique electrooptical properties and potential applications in areas ranging from nanotechnology to biotechnology, but they are challenging to synthesize. ${ }^{[1]}$ Such anisotropic nanostructures with extremely high aspect ratios can be formed when thin crystalline layers roll up under disparate surface stresses on opposite sides of the layers. ${ }^{[2,3]}$ Single-crystal nanotubes have also been synthesized using nanowire templates, ${ }^{[4]}$ and this method has been extended to prepare polymeric nanotubules with controlled diameters. ${ }^{[5]}$ Self-assembly of block copolymers and amphiphilic molecules in solution leads to the formation of tubular structures in a selective solvent that acts as a molecular structure-directing template. ${ }^{[6-8]}$ However, the self-assembly of hollow tubules without any template or solvent has not yet been realized, with few exceptions. ${ }^{[9,10]}$ Furthermore, we are not aware of any natural or synthetic self-assembling layers that spontaneously form scrolls in the solid state, which is crucial for practical applications in nanotechnology. Herein we report the formation of hollow tubules from the self-assembly of T-shaped rod-coil molecules in the solid state.

Rod-coil molecules are attractive for generating highly defined nanostructures that have physical dimensions as small as a few nanometers. ${ }^{[1]}$ The stiff rodlike components of the

[*] D.-J. Hong, E. Lee, H. Jeong, Prof. M. Lee

Center for Supramolecular Nano-Assembly and Department of

Chemistry, Yonsei University

Shinchon 134, Seoul 120-749 (Korea)

Fax: $(+82)$ 2-393-6096

E-mail: mslee@yonsei.ac.kr

Homepage: http://csna.yonsei.ac.kr

J.-K. Lee, W.-C. Zin

Department of Materials Science and Engineering

Polymer Research Institute, Pohang University of Science and

Technology, Pohang 790-784 (Korea)

T. D. Nguyen, S. C. Glotzer

Department of Chemical Engineering and Department of Materials Science and Engineering, University of Michigan

Ann Arbor, MI 48109-2136 (USA)

[**; This work was supported by the Creative Research Initiative Program of the Ministry of Education, Science and Technology of the Korean Government. D.J.H. and E.L. thank a fellowship of the BK21 program of the Ministry of Education, Science and Technology of the Korean Government. T.D.N. acknowledges the support of the Vietnam Education Foundation. T.D.N. and S.C.G. acknowledge the support of the US Department of Energy, Basic Energy Science Program and the US Air Force Office of Scientific Resesarch.

0 Supporting information for this article is available on the WWW under http://dx.doi.org/10.1002/anie.200804307. molecules endow the materials with anisotropic properties arising from their strong tendency to be aligned perpendicular to the direction of rod orientation. In addition to conventional layered structures, the rodlike components of rod-coil building blocks can be made to self-assemble into a wide variety of complex geometries, including perforated layers, ribbons, and bundles, through covalent attachment of long flexible chains to their distal section. ${ }^{[12]}$ These nanoscale objects are further organized into two-dimensional (ribbons) and three-dimensional (bundles) structures. ${ }^{[12,13]}$ However, the rod segments arrange parallel to each other to form only flat local structures as opposed to radial structures, a prerequisite for nanotubules. ${ }^{[14]}$ Herein, we report the formation of filled cylindrical and hollow tubular scrolls from the self-assembly of Tshaped rod-coil molecules in the solid state. The scrolls have a hierarchical structure comprised of alternating rod and coil layers with a narrow distribution of outer scroll diameters.

The rod-coil molecules described herein consist of a penta- $p$-phenylene conjugated rod connected to a poly(propylene oxide) (PPO) coil laterally attached through an imidazole linkage (Figure 1a). ${ }^{[1]}$ Rod-coil molecules based on lateral chains self-assemble into layered structures in which the rod segments are aligned parallel to the layer planes. ${ }^{[15,16]}$ The stiff rod layers are separated by the amorphous layers of the lateral coils. Within the layers, the rods are organized parallel to each other to form sublayers. This special organization of the rod segments into anisotropic 2D layers could be envisioned to roll up into tubules under appropriate conditions. ${ }^{[17-19]}$

Rod-coil molecules $\mathbf{1}$ and 2, and their analogues $\mathbf{3}$ and $\mathbf{4}$ with longer PPO chains, show an ordered state that is retained up to an isotropization transition (Table 1). The ordered structure is thermodynamically stable, as evidenced by differential scanning calorimetry (DSC; Table 1). The solid-state structure was confirmed by X-ray measurements (Figure $1 \mathrm{~b}$ ). Small-angle X-ray scattering (SAXS) reveals that $\mathbf{1}$ and $\mathbf{2}$ have a layered structure with a primary spacing of 3.5 and $4.6 \mathrm{~nm}$, respectively. These dimensions correspond roughly to the respective laterally extended molecular lengths, suggesting that the rod segments within the domains are parallel to the layer planes. Notably, both $\mathbf{1}$ and $\mathbf{2}$ exhibit an additional sharp reflection at an identical $q$ spacing of $3.57 \mathrm{~nm}^{-1}$ and show similar wide-angle X-ray scattering (WAXS) patterns (Figure S2 in the Supporting Information), indicating that both compounds have an essentially identical crystalline structure within the rod layers. This finding is also reflected in the similar transition temperatures and corresponding enthalpy changes (Table 1). The existence of a sharp peak at an intermediate angle suggests that the rod segments in $\mathbf{1}$ and 
a)

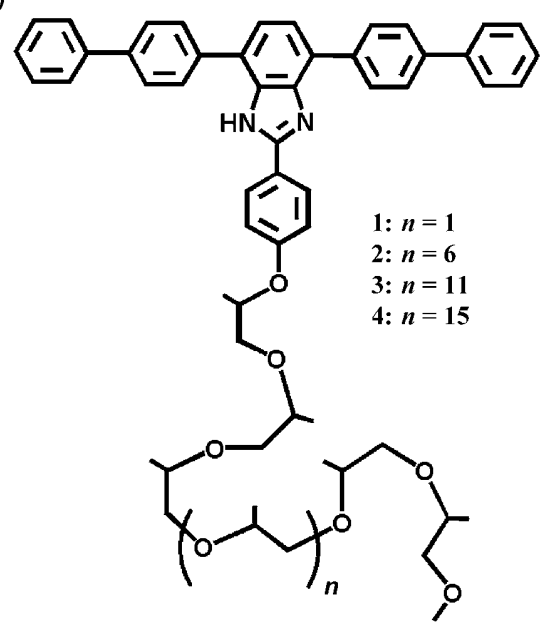

b)

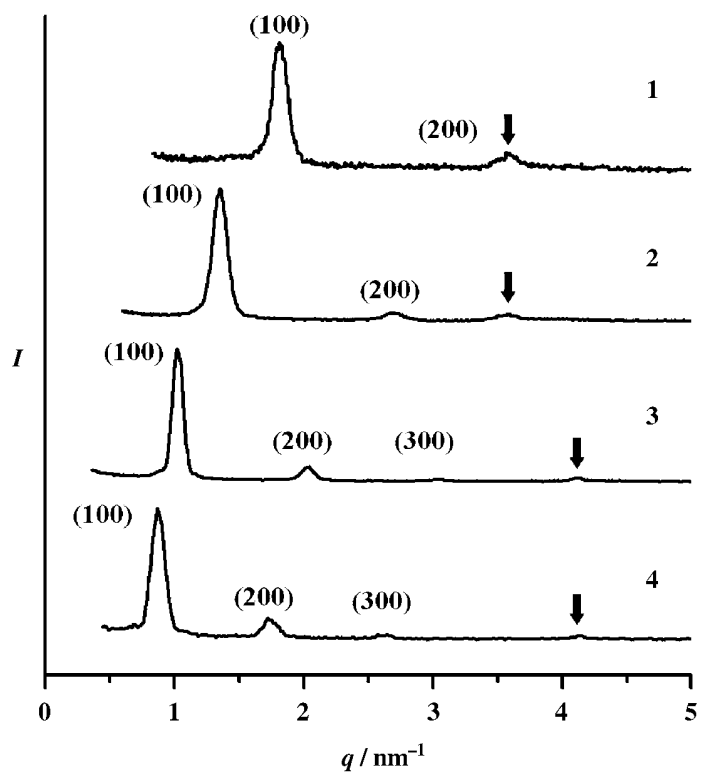

Figure 1. a) Molecular structures of 1-4. b) 1D SAXS patterns of 1-4 plotted against $q\left(=4 \pi \sin \theta \lambda^{-1}\right)$. Black arrows indicate an intermediate-angle reflection for each molecule.

Table 1: Characterization of $1-4$ by $\mathrm{X}$-ray scattering and thermal transition.

\begin{tabular}{lllll}
\hline & $\mathbf{1}$ & $\mathbf{2}$ & $\mathbf{3}$ & $\mathbf{4}$ \\
\hline$T_{\mathrm{c}}\left[{ }^{\circ} \mathrm{C}\right]^{[\mathrm{a}]}$ & 113 & 112 & 75 & 61 \\
$\left.\Delta H[\mathrm{k}] \mathrm{mol}^{-1}\right]^{[b]}$ & 16.7 & 12.0 & 5.4 & 2.3 \\
$d[\mathrm{~nm}]^{[\mathrm{c}]}$ & 3.5 & 4.6 & 6.2 & 7.2
\end{tabular}

[a] Transition temperature estimated from differential scanning calorimetry. [b] Enthalpy change $(\Delta H)$ estimated from differential scanning calorimetry. [c] Layer thicknesses (d) measured from $q$ values of 1D X-ray profiles.

2 organize into sublayers with a thickness of $1.76 \mathrm{~nm}$ within a layer. Considering the calculated rod length of $2.2 \mathrm{~nm}$ by the Corey-Pauling-Koltun (CPK) model, this dimension indi- cates that the rods are tilted relative to the sublayer normal with an angle of $37^{\circ}$. Recent simulations of laterally grafted rod-coil molecules predict the existence of in-plane sublayers with rod tilt. ${ }^{[16]}$

In contrast to conventional layered structures, these layers form scrolls with a nearly uniform diameter, as revealed by the transmission electron microscopy (TEM) images in Figure 2. The side-view image of cryomicrotomed films of $\mathbf{2}$
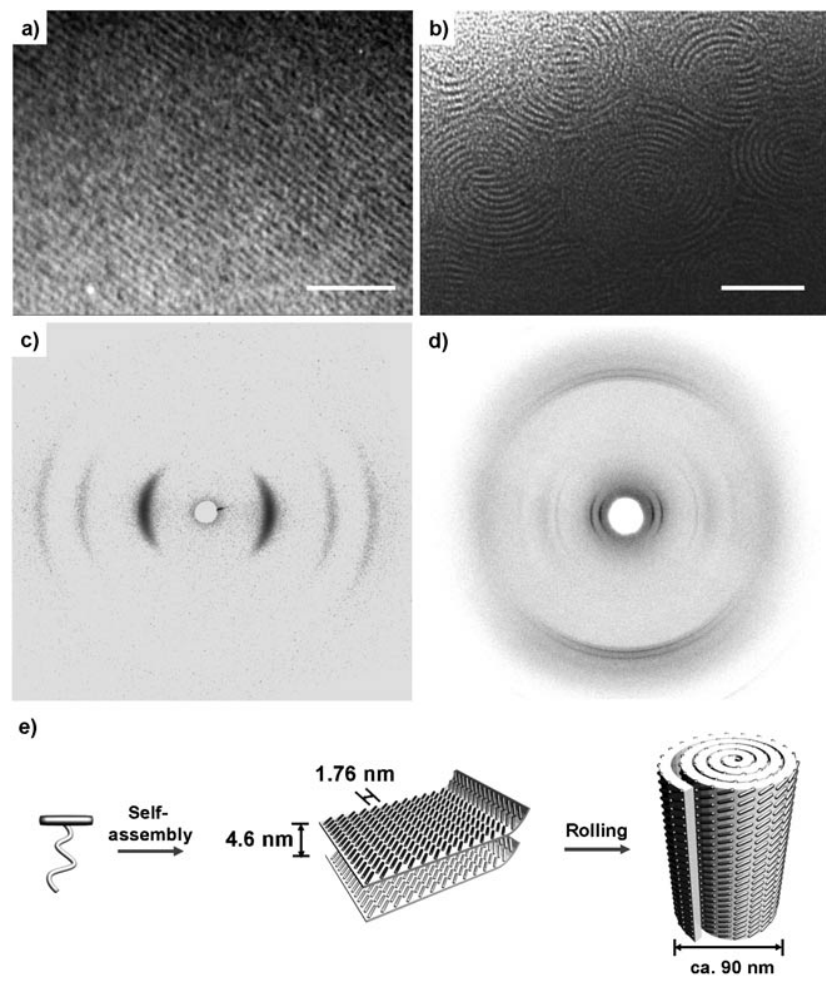

Figure 2. a) TEM image of a layered structure confined in the scrolls, revealing an ordered array of alternating light-colored PPO layers and dark aromatic layers. b) Cross-sectional image along the rolled lamellar sheet axis. Scale bars $50 \mathrm{~nm}$. c) 2D SAXS pattern obtained from 2 at $25^{\circ} \mathrm{C}$. d) $2 \mathrm{D}$ WAXS pattern of 2 . The diffraction pattern shows $\{010\}$, $\{110\}$ reflections of the rolled lamellar structure. e) Schematic representation of the filled scrolls from 2 .

stained with $\mathrm{RuO}_{4}$ shows a layered structure with a primary spacing of $4.6 \mathrm{~nm}$, consistent with that obtained from SAXS. Views of the cross-section, however, reveal the formation of scrolls with a spiral arrangement of alternating light coil and dark rod layers with scroll diameters ranging from 80 to $100 \mathrm{~nm}$ (Figure 2b). Additional information on the structural dimensionality was obtained by $2 \mathrm{D}$ X-ray diffraction with an annealed sample of $\mathbf{2}$ exhibiting macroscopic orientation. As shown in Figure 2c, the X-ray beam was directed perpendicular to the principle axes of the scrolls. At small angles, two pairs of strong (100) and (200) reflections are diffracted along the equator. At an intermediate angle, the additional repeat distance associated with the sublayer thickness in each rod layer occurs at the same direction relative to the layer reflections, demonstrating that the scroll axis is parallel to the sublayer direction. At wide angles, two pairs of reflections 
associated with the inter-rod distance are diffracted along the meridian (Figure $2 \mathrm{~d}$ ), indicative of crystallization of the rod segments within the sublayers. This result demonstrates that the repeat distance associated with $\pi-\pi$ stacking interactions is perpendicular to the rolling axis.

The electron microscopy observations together with the $\mathrm{X}$-ray results lead to the structural model of the scrolls of $\mathbf{2}$ as shown in Figure $2 \mathrm{e}$. The bulk layers are formed parallel to the molecular long axes with a thickness of $4.6 \mathrm{~nm}$, and the rod segments within the layers are organized parallel to each other to form sublayers with rod tilt with respect to the sublayer normal at an angle of $37^{\circ}$. Subsequently, the layers roll up along the direction of the sublayers to form scrolls with diameters ranging from 80 to $100 \mathrm{~nm}$. As evidenced in the simulations described below, the rod tilt with respect to the sublayer normal leads to helical scrolls, because the rolling axis is parallel to the sublayer direction.

Rod-coil molecules 3 and $\mathbf{4}$, based on longer PPO chains, also self-organize into layered structures. Each small-angle $\mathrm{X}$ ray diffraction pattern shows three sharp equidistant reflections, indicative of layered structures with lattice constants of 6.2 and $7.2 \mathrm{~nm}$ for $\mathbf{3}$ and $\mathbf{4}$, respectively (Figure $1 \mathrm{~b}$ ). These dimensions suggest that the layer structure arises from segregation of the lateral PPO chains from the stiff aromatic segments. Within the layers, the rod segments are again organized parallel to the layer planes. Furthermore, both compounds exhibit an identical intermediate angle reflection at $q=4.12 \mathrm{~nm}^{-1}$ and also show similar wide-angle X-ray diffraction patterns (Figure S2 in the Supporting Information), indicating that both molecules have an essentially identical packing structure within the rod layers. This similarity is reflected in similar transition temperatures and corresponding enthalpy changes (Table 1). The lower transition temperatures and smaller enthalpy changes compared to those of the analogues with shorter PPO chains indicate that the rod segments within the layers of $\mathbf{3}$ and $\mathbf{4}$ are aligned with overall less order than is the case for $\mathbf{1}$ and $\mathbf{2}$. The sharp intermediate-angle peak suggests that the rod segments in the two compounds organize into sublayers with a thickness of $1.52 \mathrm{~nm}$. Considering the calculated rod length of $2.2 \mathrm{~nm}$ by the CPK model, this dimension indicates that the rods are tilted with an angle of $47^{\circ}$ relative to the sublayer normal.

When cryomicrotomed films of $\mathbf{3}$ stained with $\mathrm{RuO}_{4}$ were characterized by TEM, stacks of long cylindrical objects with nearly uniform diameters of $(90 \pm 10) \mathrm{nm}$ and lengths up to several tenths of micrometers were observed (Figure 3 ). The magnified images reveal that the elongated objects consist of an inner core of approximately $30 \mathrm{~nm}$ separated by multilayer walls of about $30 \mathrm{~nm}$, demonstrating that the $1 \mathrm{D}$ objects are multiwall tubules. The image of the cross-section of the tubules shows that the walls consist of layers rolled into a spiral in which the layer thickness is $6.2 \mathrm{~nm}$ (Figure 3c), consistent with the lattice constant determined from smallangle X-ray diffraction. These results suggest that the formation of a tubular structure arises from a rolling up of the layers. When thin films of $\mathbf{3}$ were cast from dilute methanol solutions and negatively stained with uranyl acetate solution, which is able to fill the empty space by capillary action, the image showed a dark interior in the elongated
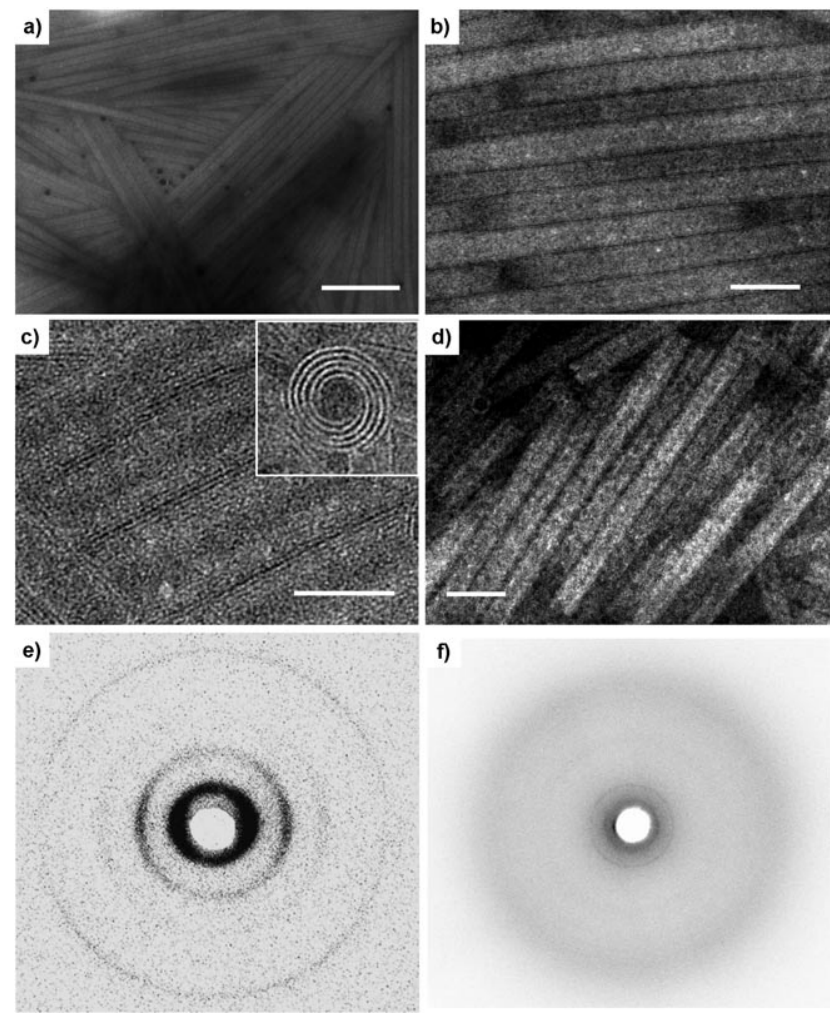

Figure 3. a) Low-magnification TEM image of a microtomed film of $\mathbf{3}$ showing long cylindrical aggregates with length up to several micrometers. Scale bar $1 \mu \mathrm{m}$. b, c) Close examination of the same film reveals a multilayered tubular structure with an interlayer distance of the tube wall of about $6.2 \mathrm{~nm}$. The inset in (c) is a high-magnification image of the cross-section; it shows the rolled layers with a spiral arrangement, implying that the tubes are composed of rolled-up sheets in a layered structure. Scale bars 200 (b) and $100 \mathrm{~nm}$ (c). d) TEM image of a solution-cast thin film of $\mathbf{3}$ negatively stained with uranyl acetate, also revealing the formation of a well-ordered tubular structure. The light regions correspond to the tube wall, the dark regions to the inner cavity of the tube containing the staining agent. Scale bar $200 \mathrm{~nm}$. e) 2D-SAXS pattern obtained from 3 at $25^{\circ} \mathrm{C}$. The diffraction pattern shows $\{100\},\{200\}$, and $\{300\}$ reflections. f) $2 D$ WAXS pattern of 3 . The diffraction pattern shows a broad halo in the wide-angle region.

objects (Figure $3 \mathrm{~d}$ ), clearly indicating a hollow cavity along the scroll axis.

To investigate the molecular orientation within the rolled tubules, 2D X-ray scattering experiments were performed with an annealed sample of $\mathbf{3}$. The diffraction pattern shows two pairs of strong (100) and (200) reflections that are diffracted along the equator at small angles (Figure $3 \mathrm{e}$ ). At intermediate angles, two pairs of diffraction arcs corresponding to a sublayer thickness are found in the cross directions at an angle of approximately $90^{\circ}$, implying that the rods are packed into a smectic $\mathrm{C}$ liquid-crystal-like structure with a tilt angle of approximately $45^{\circ}$ relative to the sublayer normal. At wide angles, a diffuse scattering centered at $0.45 \mathrm{~nm}$ is diffracted in a circular pattern (Figure $3 \mathrm{f}$ ), characteristic of liquid-crystalline order between the rod segments.

To determine whether the tubules form directly from the melt or by spontaneous rolling up of planar sheets, we 
investigated cryomicrotomed films of $\mathbf{3}$ with different annealing times (Figure $4 \mathrm{a}-\mathrm{d}$ ). When the melt was quickly quenched by blowing cold argon onto it, the TEM images showed
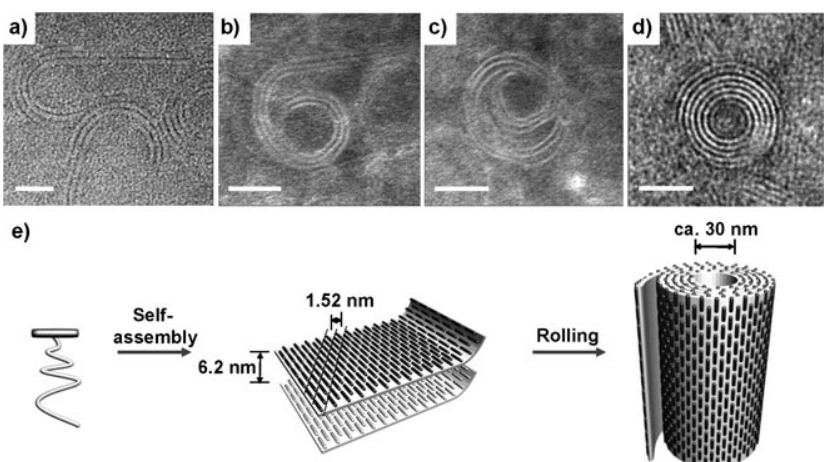

Rolling

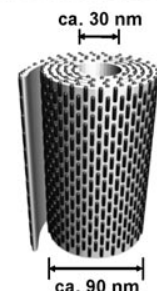

Figure 4. TEM micrographs of intermediate structures kinetically trapped by quick quenching from the melt of 3. TEM analysis was performed on thin films cryomicrotomed at $-70^{\circ} \mathrm{C}$. TEM images of the cross-sections from the experiment show the intermediate structures between lamellar sheets and tubular scrolls. The sheets bend slightly (a), then roll upon themselves $(b, c)$ to form the tubular scrolls (d). Scale bars $50 \mathrm{~nm}$. e) Schematic representation of the tubular scrolls of 3 .

loosely curved layers together with uncurved layers. However, when the films were cooled slowly from the melt to ambient temperature, we observed closely packed scrolls throughout the image area (Figure $4 \mathrm{~d}$ ). This result implies that the molecules first assemble into a layered structure that subsequently forms scrolls. On the basis of these results, we hypothesize that the hollow tubules are formed by rolling up along an axis parallel to the rod direction of the bulk layers in which the laterally grafted rod segments are arranged parallel to each other to form sublayers with a rod tilt of approximately $45^{\circ}$ relative to the sublayer normal (Figure $4 \mathrm{e}$ ). These scrolls have external diameters ranging from $80-100 \mathrm{~nm}$ and a hollow interior with a diameter of approximately $30 \mathrm{~nm}$.

To gain further insight into the rolling behavior of the layers of T-shaped rod-coil molecules, we performed Brownian dynamics simulations (for details, see the Supporting Information) of individual preformed bilayer sheets of molecules $\mathbf{1}$ or $\mathbf{2}$ and $\mathbf{3}$ or $\mathbf{4}$. Our previous simulations ${ }^{[16]}$ demonstrated the formation of these sheets from the selfassembly of T-shaped rod-coil building blocks under melt conditions, but our simulation approach did not permit their subsequent assembly into higher-order structures such as scrolls. We used experimentally determined values to set the length and spacing of the rods and the coils relative to the layer thickness. Flat sheets of $\mathbf{1}$ and $\mathbf{2}$ with various aspect ratios (see the Supporting Information) were used in the simulations with the rods organized into crystalline, in-plane sublayers with a rod tilt angle of $37^{\circ}\left(P_{2}\right.$ symmetry $\left.{ }^{[16]}\right)$, as shown in Figure $2 \mathrm{e}$. The packing of rods within the sheet was maintained by a bonding scheme as illustrated in Figure S4a (in the Supporting Information) to mimic the expected strong $\pi-\pi$ stacking between neighboring aligned rods and weaker end-to-end interactions. After initialization and subsequent relaxation of the sheets (see the Supporting Information), we observed the spontaneous rolling of sheets, regardless of aspect ratio (Figure $5 \mathrm{a}$, top row and bottom left image). The a)

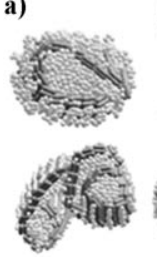

c)

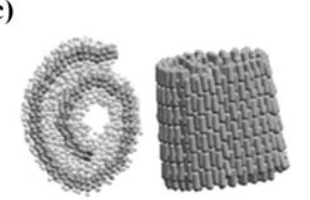

b)

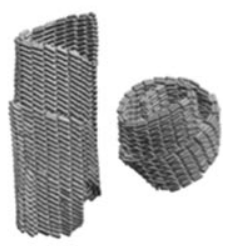

Figure 5. Simulation results. a) Top-view snapshots of scrolls formed by sheets for which the rod packing is as proposed in Figure 2 e for 1 and $\mathbf{2}$ with different aspect ratios (measured in pairs of rods), rod tilt angles, and short and long coil lengths, corresponding to 1 ("short" coil) and 2 ("long" coil). Upper row: (48:12, 37", short coil), (60:8, $37^{\circ}$, long coil), and $\left(48: 8,37^{\circ}\right.$, long coil). Lower row: $\left(60: 12,37^{\circ}\right.$, short coil), (48:12, $0^{\circ}$, short coil), and (48:12, $0^{\circ}$, long coil). b) Side-view snapshots of scrolls formed by sheets with different rod tilt angles in which the rod packing is as proposed in Figure $2 \mathrm{e}$ for $\mathbf{2}$ (coils removed for clarity). Left: (48:12, 37\%, long coil). Right: (48:12, $0^{\circ}$, long coil). c) Snapshots of scrolls formed by sheets for which the rod packing is as proposed for $\mathbf{3}$ and $\mathbf{4}$ in Figure $4 \mathrm{e}$. The first two images from the left are the top view and side view (with coils removed) of a 6:80 sheet with short coils corresponding to compound 3 . The third image shows the top view of a scroll formed from a 6:80 sheet with long coils corresponding to compound 4 .

simulations confirm that the rolling axis is parallel to the sublayer direction for all aspect ratios studied (Figure 2e). Top-view snapshots of the simulated structures show excellent agreement with the TEM images of compound 2 (Figure $2 b$ ), in which cylindrical scrolls are formed with alternating layers of rods and coils and have a coil-filled center. Simulations of bilayers with zero rod tilt angle also yield cylindrical scrolls (Figure $5 \mathrm{a}$, bottom middle and right). The side-view snapshots of the simulated structures (Figure $5 \mathrm{~b}$ ) indicate that the scrolls are helical for nonzero tilt angles. We see that the $P_{2}$ crystalline packing of the rods in the sheet is well maintained during rolling (Figure $5 \mathrm{~b}$ ) owing to the bonding scheme, thus demonstrating the importance of the strong $\pi-\pi$ interactions between the rods in the sublayers to achieving the observed scrolls.

To elucidate the formation of the tubular scrolls, we simulated bilayer sheets of molecules of $\mathbf{3}$ and $\mathbf{4}$, assuming that the rods are arranged in loosely packed, liquid crystalline sublayers with a rod tilt angle of $45^{\circ}$ (similar to $C_{\mathrm{mm}}$ packing ${ }^{[16]}$ ), as shown in Figure 4 e. Again, sheets of various aspect ratios (Figure S4b in the Supporting Information) were initialized, relaxed, and allowed to fluctuate dynamically, which led to their spontaneous rolling. Our simulations (Figure $5 \mathrm{c}$ ) confirm that the rolling axis is parallel to the 
rod direction in every case, consistent with the model proposed in Figure $4 \mathrm{e}$. The simulated structures (Figure 5c, left) show excellent agreement with the hollow tubules formed by compounds 3 and $\mathbf{4}$. We observe that the loose packing of the rods in interdigitated sublayers is maintained under rolling (Figure 5c, middle). Furthermore, our simulations reveal that the radius of the hollow core increases with the coil length (Figure $5 \mathrm{c}$, right), presumably because of the limited compression of the coils and the increasing thickness (and thus decreasing bending elasticity) of the bilayer sheets.

The results described herein demonstrate that T-shaped rod-coil molecules self-assemble into layered structures in which the long axis of the rod segment is parallel to the layer plane. The layers, in turn, unexpectedly roll up to form scrolls in the solid state. Rolling of the layers can be understood by considering the grafting density at the rod-coil interface and the resulting space-filling requirements. Lamellar ordering of rods would confine rod-coil junctions to a flat interface. However, the large mismatch between the cross-sectional areas of the coil segments and the side face of the rod results in a strong energetic penalty associated with low grafting density at the planar rod-coil interface. As a result, the flat 2D rod layer curves along the direction of the sublayer ( $\mathbf{1}$ and $\mathbf{2}$ ) or the rod axis ( $\mathbf{3}$ and $\mathbf{4}$ ) and ultimately rolls with the coil layer inside to release the surface stresses. ${ }^{[20]}$

In contrast to $\mathbf{1}$ and $\mathbf{2}$, which curve into filled cylindrical scrolls, 3 and $\mathbf{4}$ (which display longer PPO chains) form tubular scrolls with hollow channels. This remarkable contrast in scroll structure with small variation in coil length of the rod-coil molecule can be explained in terms of the packing arrangements of the rod segments within the layers. In the case of $\mathbf{1}$ and $\mathbf{2}$, the rolling axis is parallel to the sublayer direction. Strong stacking interactions of the rod segments caused by shorter laterally grafted coils, as reflected in the wide-angle X-ray diffraction patterns, prevent bending along the rod direction, whereas relatively weak end-to-end interactions between rods in adjacent sublayers result in bending at each sublayer separation to form tightly packed scrolls. In contrast, the rolling axis of $\mathbf{3}$ and $\mathbf{4}$ is parallel to the rod axis, which is attributed to relatively loose packing of the rod segments and the resulting poorly defined sublayers arising from the longer grafted coils. The loose packing of the rod segments is supported by wide-angle $\mathrm{X}$-ray diffraction patterns showing a diffuse halo centered at $0.45 \mathrm{~nm}$. However, the tightness of the scroll along the rod direction is limited by the tendency for the sheet to retain energetically favorable inter-rod interactions and by the steric congestion of long coils inside the tubules. This limited bending elasticity of the rod layer leads to the formation of a central hole in the scrolls. ${ }^{[21]}$
The most notable feature of the structurally simple rodcoil molecules investigated herein is their ability to hierarchically and reversibly self-assemble into scrolls in the solid state. It is also remarkable that the core structure of the scrolls, from filled cylindrical to hollow tubular, can be controlled by only a small variation of coil length of the T-shaped rod-coil molecule. Such solid-state scrolls with controlled interior hold outstanding potential for a broad range of applications in nanoscience.

Received: September 1, 2008

Published online: December 12, 2008

Keywords: amphiphiles - liquid crystals - nanostructures · self-assembly

[1] G. R. Patzke, F. Krumeich, R. Nesper, Angew. Chem. 2002, 114, 2554; Angew. Chem. Int. Ed. 2002, 41, 2446.

[2] R. Tenne, Nat. Nanotechnol. 2006, 1, 103.

[3] O. G. Schmidt, K. Eberl, Nature 2001, 410, 168.

[4] J. Goldberger, R. He, Y. Zhang, S. Lee, H. Yan, H.-J. Choi, P. Yang, Nature 2003, 422, 599.

[5] M. Steinhart, J. H. Wendorff, A. Greiner, R. B. Wehrspohn, K. Nielsch, J. Schilling, J. Choi, U. Gösele, Science 2002, 296, 1997.

[6] G. C. L. Wong, J. X. Tang, A. Lin, Y. Li, P. A. Janmey, C. R. Safinya, Science 2000, 288, 2035.

[7] J. P. Hill, W. Jin, A. Kosaka, T. Fukushima, H. Ichihara, T. Shimomura, K. Ito, T. Hashizume, N. Ishii, T. Aida, Science 2004, 304, 1481.

[8] D. T. Bong, T. D. Cark, J. R. Granja, M. R. Ghadiri, Angew. Chem. 2001, 113, 1016; Angew. Chem. Int. Ed. 2001, 40, 988.

[9] M. R. Ghadiri, J. R. Granja, R. A. Milligan, D. E. McRee, N. Khazanovich, Nature 1993, 366, 324.

[10] V. Percec, A. E. Dulcey, V. S. K. Balagurusamy, Y. Miura, J. Smidrkal, M. Peterca, S. Nummelin, U. Edlund, S. D. Hudson, P. A. Heiney, H. Duan, S. N. Magonov, S. A. Vinogradov, Nature 2004, 430, 764.

[11] M. Lee, B.-K. Cho, W.-C. Zin, Chem. Rev. 2001, 101, 3869.

[12] J.-H. Ryu, N.-K. Oh, W.-C. Zin, M. Lee, J. Am. Chem. Soc. 2004, 126, 3551.

[13] W.-Y. Yang, J.-H. Ahn, Y.-S. Yoo, N.-K. Oh, M. Lee, Nat. Mater. 2005, 4, 399.

[14] M. A. Horsch, Z. Zhang, S. C. Glotzer, Phys. Rev. Lett. 2005, 95, 056105 .

[15] C. Tschierske, Chem. Soc. Rev. 2007, 36, 1930.

[16] M. A. Horsch, Z. Zhang, S. C. Glotzer, Nano Lett. 2006, 6, 2406.

[17] L. Radzihovsky, J. Toner, Phys. Rev. Lett. 1995, 75, 4752.

[18] M. Bowick, M. Falcioni, G. Thorleifsson, Phys. Rev. Lett. 1997, 79,885 .

[19] M. Bowick, A. Travesset, Phys. Rev. E 1999, 59, 5659.

[20] T. M. Birshtein, P. A. Iakovlev, V. M. Amoskov, F. A. M. Leermakers, E. B. Zhulina, O. V. Borisov, Macromolecules 2008, 41, 478.

[21] B. Lotz, S. Z. D. Zheng, Polymer 2005, 46, 577. 